Aurélien Lignereux, Gendarmes et policiers dans la France de Napoléon. Le duel Moncey-Fouché, préface de Jean Tulard, Maisons-Alfort, Service historique de la Gendarmerie nationale, Collection Études, 2002, 275 p. ISBN : 2110936436.16 euros.

Laurent Lopez

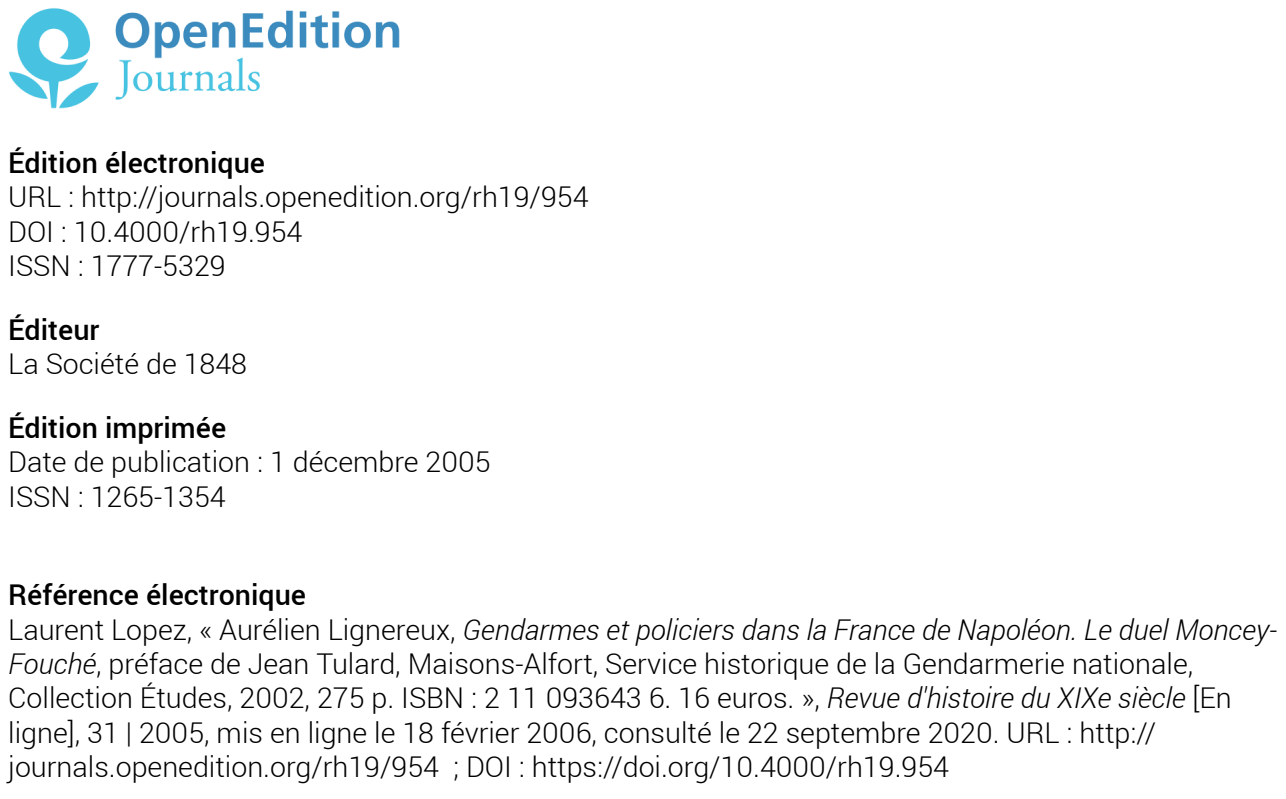

Ce document a été généré automatiquement le 22 septembre 2020.

Tous droits réservés 


\title{
Aurélien Lignereux, Gendarmes et policiers dans la France de Napoléon. Le duel Moncey-Fouché, préface de Jean Tulard, Maisons-Alfort, Service historique de la Gendarmerie nationale, Collection Études, 2002, 275 p. ISBN : 2110936436.16 euros.
}

\author{
Laurent Lopez
}

1 Les policiers et les gendarmes qui écrivent l'histoire de leurs corps respectifs en font presque systématiquement remonter les origines au Moyen Âge, voire à l'Antiquité. La dualité des forces de l'ordre en France serait, par conséquent, multiséculaire, peut-être millénaire, à en croire ces auteurs. La rivalité de la police et de la gendarmerie, qui ressurgit régulièrement depuis le début du $\mathrm{XIX}^{\mathrm{e}}$ siècle - de même que les débats politiques pour tenter de l'apaiser - épouse-t-elle cette ancienneté ? L'étude d'Aurélien Lignereux a l'ambition de contribuer à la compréhension des «origines du vieux contentieux opposant gendarmes et policiers ». L'actualité gouvernementale confère donc à ce livre une pertinence qui fait ressortir toutes les qualités d'une maîtrise menée sous la direction de Jean-Noël Luc à l'Université Paris IV-Sorbonne et distingué par le prix littéraire de la gendarmerie en 2001.

2 La France de Napoléon, autrement dit le Consulat puis l'Empire des cent trente départements, constitue la matrice de l'antagonisme de deux institutions qui naissent et se consolident alors. Le prisme du «duel » entre le ministre de la Police générale, Joseph Fouché, et le premier inspecteur général de la Gendarmerie, le maréchal BonAdrien Janot de Moncey, permet de mettre en perspective le panorama de rivalités s'exprimant à de multiples échelles, du sommet de l'État aux niveaux hiérarchiques les 
plus bas. L'auteur s'attache particulièrement à la période 1804-1810, c'est-à-dire celle du second passage de Fouché à la tête de la Police générale. Il construit, à partir de textes réglementaires, lettres, rapports, une " histoire relationnelle ", dont le sujet est cet « entre-deux » d'une histoire proprement policière ou spécifiquement dédiée à la gendarmerie. Sa démarche procède en deux temps : il présente d'abord le déroulement chronologique de la rivalité entre militaires et policiers puis il réorganise thématiquement cette première structure. Il analyse le cadre réglementaire, puis les querelles participant d'une guerre des représentations entre deux corps dont les identités émergentes se cristallisent en s'opposant et, enfin, donne des exemples des affrontements nés de leurs rapports directs sur le terrain.

3 Avant d'être une opposition entre des personnes, les relations entre policiers et gendarmes sont d'abord une question de textes. Le 2 janvier 1796 est institué le ministère de la Police générale. La gendarmerie, pourtant antérieure de cinq années, est placée sous sa tutelle pour ses principales attributions en matière de police administrative (la prévention des infractions et des troubles à l'ordre public) et de police judiciaire (la recherche des auteurs des crimes et des délits). Cette dépendance est réaffirmée par la loi organique du 17 avril 1798 fixant ses missions. Ainsi son existence, presque dès son origine, est soumise à une contradiction grosse des affrontements ultérieurs avec les policiers. Appartenant à un corps qui se définit par son caractère militaire, les gendarmes dépendent essentiellement de civils - ministre de la Police, ministre de la Justice, préfets, commissaires - pour l'accomplissement de la majorité de leurs tâches. C'est, néanmoins, la nomination, en décembre 1801, du maréchal Moncey à la tête de la récente gendarmerie qui inaugure - révèle ? l'antagonisme entre policiers et gendarmes, à commencer par leurs chefs respectifs. Alors que la coexistence entre le colonel Radet, nommé inspecteur général de la Gendarmerie en mars 1800, et Fouché, qui prend la tête de la Police générale en juillet 1799, se déroule sans anicroche notable, Moncey s'efforce d'éviter que la dépendance de la gendarmerie ne devienne subordination pure et simple grâce aux manœuvres du duc d'Otrante. Napoléon est à la fois l'ombre tutélaire pesant sur les deux hommes et l'arbitre de leurs prétentions, l'un à une autonomie vis-à-vis du ministère de la Police générale, l'autre à une mainmise directe sur les gendarmes couvrant l'ensemble du territoire impérial. Le «duel Moncey-Fouché » tourne d'abord à l'avantage du premier lorsque, le 15 septembre 1802, est supprimé le ministère du second. Le champ laissé libre par ce départ forcé accroît l'étendue des missions dévolues aux gendarmes et accomplies peu de temps avant par les policiers. Aurélien Lignereux montre bien que l'image d'une gendarmerie s'interdisant les "missions occultes", formellement proscrites par les décrets organiques de 1854 et 1903, n'est pas de mise pour le début du XIX ${ }^{e}$ siècle, contrairement à une représentation interne au corps, née sous la Second Empire et constamment invoquée sous la Troisième République. La description de la répression des menées contre-révolutionnaires révèle des pratiques qui contrastent avec la mémoire d'une institution construite durant le XIX ${ }^{e}$ siècle ; celle-ci réserve, du moins dans ses discours, les basses œuvres de la haute police aux Vidocq de la préfecture de police sous la Monarchie de Juillet ou aux commissaires de la police spéciale des chemins de fer de la Sûreté générale à partir de la deuxième moitié du XIX ${ }^{\mathrm{e}}$ siècle. Si l'auteur dévoile des gendarmes accomplissant des enquêtes de police secrète, il insiste également sur ces militaires qui quittent leur uniforme et revêtent des déguisements pour faciliter leurs recherches et l'appréhension de malfaiteurs. Les débats soulevés par des procédés qui effacent les différences avec les policiers agissant 
en civil témoignent, a contrario, de l'importance des signes distinctifs et identitaires que cherchent à s'approprier chacun des acteurs de ces institutions. Au-delà des méthodes employées par les gendarmes pour accomplir ces missions, ce qui pose problème réside dans la nature même des missions effectuées. À une époque où les deux institutions émergentes défendent, par des tentatives réciproques de discrédit, la légitimité de leur existence, la délimitation de compétences exclusives pour chacune des deux forces de police est un enjeu crucial. Il est difficile de ne pas songer à la récurrence, jusqu'à aujourd'hui, de la question du port de l'uniforme en matière de police judiciaire. Si l'un des mérites essentiels de l'ouvrage d'Aurélien Lignereux est de participer à la compréhension de la genèse de la rivalité entre la gendarmerie et l'institution policière, son étude n'en est pas moins fondamentale pour saisir, à leur origine, des enjeux traversant le corps militaire pendant les deux derniers siècles.

Avec le rétablissement du ministère de la Police générale, dirigé à nouveau par Fouché, en juillet 1804, le conflit entre policiers et gendarmes, tout en retrouvant son acuité, se déroule désormais au détriment des gendarmes. Le point culminant de cette " guerre des bureaux » est atteint avec la crise des mois de février et mars 1805. Moncey réagit vivement à une directive adressée au préfet de la Marne par le conseiller d'État Miot. Alors que celui-ci, soutenu par Fouché, conçoit les rapports entre police et gendarmerie en termes d'instrumentalisation de la seconde par la première, Moncey continue à défendre auprès du souverain l'autonomie et la spécificité militaire du corps. Cet épisode montre que la dualité des forces de l'ordre impérial est, si ce n'est voulue par l'empereur, en tout cas savamment entretenue pour asseoir son pouvoir personnel, au détriment de l'efficacité opérationnelle qui pourrait résulter d'une coopération accrue entre militaires et policiers. Napoléon $I^{\text {er }}$ trouve dans la gendarmerie un utile contrepouvoir à l'ambition de Fouché, alors que l'existence de la police évite que les militaires ne contrôlent l'ensemble du dispositif, intérieur et extérieur, de "violence légitime " (Max Weber) de l'État. Née sous la Révolution et développée sous l'Empire, l'originalité d'un système policier dual, si elle fut parfois remise en cause par les républicains, ne fut néanmoins jamais abolie; sa justification changeant seulement de registre et s'appuyant, depuis la Troisième République, sur l'argument démocratique.

5 Le second ministère Fouché (juillet 1804-juin 1810) n'en marque pas moins une "cohabitation malaisée, mais acceptée" entre les tenants des deux institutions. Moncey est sur la défensive; il multiplie les gages de soumission au souverain et tente toujours de placer la gendarmerie dans une position d'« auxiliaire" plutôt que d'«instrument » du ministère de la Police générale. De son côté, Fouché change de stratégie ; il met désormais en œuvre une politique d'influence sur le corps plutôt que de tenter un contrôle direct. Deuxième motif de l'apaisement du conflit des institutions : leur "spécialisation fonctionnelle ». Les missions de haute police échoient à présent clairement aux effectifs commandés par Fouché, quand les missions de maintien de l'ordre incombent aux gendarmes. Cette division du travail policier, si elle participe au modus vivendi des rapports entre agents civils de l'ordre et policiers bottés, a une cause structurelle : l'insuffisance numérique chronique des hommes du ministère de la Police générale; un problème qui s'est prolongé au moins jusqu'à la fin de la Troisième République. Il y a là un caractère fondamental des relations entre policiers et gendarmes durant tout le XIX ${ }^{e}$ siècle, sur lequel l'auteur n'insiste pas assez : en dépit de leurs différences, de leurs rivalités, les deux institutions ne peuvent se passer l'une de l'autre. En effet, seuls les gendarmes, en raison de leur nombre, sont en mesure d'accomplir la plupart des missions relevant également de l'autorité des commissaires 
de police; dans la plupart des cas, ces derniers doivent requérir ces militaires pour rendre effectives leurs compétences. Parallèlement, l'action des gendarmes est dépendante de l'autorité des préfets et de leurs auxiliaires, les commissaires, dont l'auteur rappelle que les attributions furent fixées par le décret du 10 septembre 1805. Le duel désormais à fleurets mouchetés entre Moncey et Fouché s'explique, enfin, par l'évolution de leurs préoccupations de carrière. Les institutions cessent d'être des enjeux personnels de promotion pour leurs chefs respectifs, qui orientent désormais leurs ambitions vers d'autres horizons que l'attachement à des prérogatives étroites. Le " grondement de la bataille» (Michel Foucault) des chefs peut s'apaiser.

6 L'une des réussites de cette étude est de chercher à voir si la "guerre des bureaux » parisiens trouve un retentissement au niveau local. Le titre de l'ouvrage est à cet égard trop réducteur puisqu'il occulte l'une de ses parties les plus originales: la mise en perspective des relations entre policiers et gendarmes sur le terrain, ouvrant ainsi la voie à des recherches sur les périodes postérieures. Les réquisitions adressées par les commissaires de police aux officiers de gendarmerie constituent les motifs les plus fréquents de friction. Les questions d'amour-propre entrent pour une grande part dans les refus de militaires qui rechignent à plier devant l'autorité civile. Les représentations mutuelles traversant chacune des deux institutions sont alors essentielles pour comprendre les motivations de ces refus ou, inversement, les agacements des policiers devant la culture militaire des gendarmes. Il y a bien alors un rapport de force symbolique qui se joue à travers des représentations qui stigmatisent l'autre institution et ses pratiques. Les rapports des commissaires sur les gendarmes et les comptes rendus des gendarmes sur les actions des policiers témoignent d'une "hétérologie " (Michel de Certeau) - d'un discours de l'autre, et sur l'autre - qui se met en place pour, ensuite, se consolider durant le XIX ${ }^{\mathrm{e}}$ sècle. Néanmoins, Aurélien Lignereux relate d'heureux exemples qui nuancent le tableau d'une éternelle et irréductible querelle entre policiers et gendarmes. Il met à mal l'image de rapports inévitablement conflictuels entre les deux forces de l'ordre qui sert de grille d'analyse à des relations trop facilement résumées sous l'expression commode de "guerre des polices ». Pardelà les méfiances réciproques, les hostilités latentes entre gendarmes et policiers, les dangers de missions accomplies en commun atténuent les rivalités et scellent une estime mutuelle.

$7 \quad$ L'ultime moment de l'affrontement entre Fouché et Moncey est marqué par l'éviction du duc d'Otrante en 1810 et son remplacement par Savary, ancien chef de la légion d'élite, donc ancien gendarme. Le duel semble alors définitivement remporté par le premier inspecteur général de la gendarmerie et la police paraît devoir être "gendarmisée ", ainsi qu'en fait l'hypothèse Aurélien Lignereux. Or, l'inverse se produit : c'est justement le commandement passé de Savary dans la gendarmerie qui permet à Savary de supplanter Moncey. Profitant de son réseau d'amitiés parmi les officiers du corps, le nouveau ministre de la Police générale multiplie les opérations associant policiers et gendarmes et réduit par là leurs antagonismes. Suffit-il de faire travailler ensemble des hommes pour que cessent leurs rivalités? L'auteur le laisse penser. Que deviennent alors les représentations mutuelles stigmatisant l'autre institution? Se transforment-elles en même temps que les manœuvres de Savary? Les épisodes de coopération sur le terrain ont-elles une influence notable sur ces images? On voudrait en savoir plus. 
8 Moncey, habité par les rancœurs accumulées contre Fouché, ne change pas de position et perd le pouvoir qu'il avait sur la gendarmerie, victime également, il est vrai de la fin chaotique de l'Empire en 1815. Dans la monarchie restaurée, la gendarmerie est victime du zèle qu'elle aurait mis à accomplir les missions du despote. Avec la fin du régime napoléonien, l'institution traverse une crise qui engage son existence en raison de sa loyauté passée.

Aurélien Lignereux mène, au total, une étude rigoureuse des causes d'une rivalité perdurant jusqu'à aujourd'hui en parcourant l'ensemble des sources disponibles. Il montre l'émergence d'une division du travail policier qui s'est développée jusqu'à la fin de la Troisième République, même si elle ne s'y réduit pas. Il éclaire l'histoire de la gendarmerie du XIX en insistant sur l'accomplissement accepté pendant la période napoléonienne de missions ensuite refusées par les républicains, notamment les tâches à caractère politique. Il touche aux marges de la sociologie de l'État en analysant la naissance de la spécificité de l'appareil policier français, partagé entre forces militaires et autorités civiles. Les liens soulignés entre les institutions et les hommes qui en appliquent les règlements donnent à sa démarche une empreinte de sociologie historique. Il montre, enfin, que l'étude de la police sans celle de la gendarmerie, et inversement, revient à laisser dans l'ombre et à ne pas comprendre une partie des motivations de l'action des agents de chacune des deux forces de l'ordre et leurs interactions. 